\title{
Avaliação do conhecimento de estudantes de Odontologia sobre a atuação do Cirurgião-Dentista nas Unidades de Terapia Intensiva e Hospitalar
}

\author{
Evaluation of Knowledge of Dentistry students about the role of the Dental Surgeon in Intensive Care and \\ Hospital Units \\ Evaluación de conocimientos de estudiantes de Odontología sobre el rol del Cirujano Dentista en Unidades \\ de Cuidados Intensivos y Hospitalario
}

Recebido: 03/07/2021 | Revisado: 08/07/2021 | Aceito: 09/07/2021 | Publicado: 21/07/2021

\author{
Dandara Damasceno Holanda \\ ORCID: https://orcid.org/0000-0001-9724-9387 \\ Universidade Paulista, Brasil \\ E-mail: dandaradholanda@gmail.com \\ Leonardo Sales Falcão \\ ORCID: https://orcid.org/0000-0003-4811-339X \\ Universidade Paulista, Brasil \\ E-mail: leosalesfalcao70@gmail.com \\ Nely Cristina Medeiros Caires \\ ORCID: https://orcid.org/0000-0003-3094-4064 \\ Universidade Paulista, Brasil \\ E-mail: nelycaires@gmail.com
}

\begin{abstract}
Resumo
A Odontologia Hospitalar compreende a inserção do odontólogo na equipe multiprofissional com o objetivo de: prevenção, o diagnóstico e o tratamento de manifestações bucais e seu impacto na condição sistêmica de pacientes internados. Este trabalho avaliou o conhecimento de acadêmicos de Odontologia em MANAUS/AM, sobre a atuação do cirurgião-dentista em ambiente hospitalar. Trata-se de um estudo observacional, transversal, desenvolvido no período de janeiro a março de 2020. Participaram do estudo 80 acadêmicos, regularmente matriculados entre o $1^{\circ}$ ao $8^{\circ}$ períodos que se dispuseram a participar voluntariamente do estudo e responder ao questionário, após a leitura e assinatura do Termo de Consentimento Livre e Esclarecido (TCLE). Essa pesquisa foi aprovada pelo CEP parecer CAAE:28581020.6.0000.5512. Os resultados mostraram que 50\% dos acadêmicos nunca vivenciaram a experiência em ambiente hospitalar durante o curso de Graduação, embora mais da metade (64\%) dos entrevistados tenham demonstrado interesse por esta experiencia. Verificou-se que $41 \%$ consideram que esse conteúdo deveria ser abordado na Grade Curricular, apesar de já terem ouvido falar sobre o assunto em aulas ministradas por docente do curso. 54\% dos acadêmicos responderam que não se sentem capacitados para trabalhar junto a uma equipe médica e multiprofissional em ambiente hospitalar, embora $65 \%$ dos alunos acreditam que é importante a atuação do cirurgiãodentista nesta equipe. Conclui-se que, apesar da ausência da disciplina sobre Odontologia Hospitalar no curso de Graduação em Odontologia, existe o interesse na grande maioria dos acadêmicos sobre a odontologia hospitalar. Sugere-se implementação de disciplina na grade curricular voltada para odontologia hospitalar.
\end{abstract}

Palavras-chave: Assistência odontologica; Equipe hospitalar de odontologia; Estudantes de odontologia, Ensino.

\begin{abstract}
Hospital Dentistry comprises the insertion of dentists in the multidisciplinary team with the objective of prevention, diagnosis and treatment of oral manifestations and their impact on the systemic condition of hospitalized patients. This work evaluated the knowledge of Dentistry students in MANAUS/AM about the role of dentists in a hospital environment. This is an observational, cross-sectional study, developed from January to March 2020. The study included 80 students, regularly enrolled between the $1^{\text {st }}$ and $8^{\text {th }}$ periods, who were willing to voluntarily participate in the study and answer the questionnaire, after reading and signing the Informed Consent Form (TCLE). This research was approved by CEP opinion CAAE: 28581020.6.0000.5512. The results showed that 50\% of the students had never experienced the experience in a hospital environment during the Undergraduate course, although more than half $(64 \%)$ of the interviewees showed interest in this experience. It was found that $41 \%$ believe that this content should be addressed in the Curriculum Framework, despite having already heard about the subject in classes taught by the course's professor. 54\% of students responded that they do not feel qualified to work with a medical and multidisciplinary team in a hospital environment, although $65 \%$ of students believe that the role of dentists in this team is important. It is concluded that, despite the absence of the discipline on Hospital Dentistry in the
\end{abstract}


Undergraduate Dentistry course, there is interest in most academics about hospital dentistry. It is suggested to implement a discipline in the curriculum focused on hospital dentistry.

Keywords: Dental care; Dental staff hospital; Students dental; Teaching.

\section{Resumen}

La Odontología Hospitalaria comprende la inserción de los odontólogos en el equipo multidisciplinario con el objetivo de: prevención, diagnóstico y tratamiento de las manifestaciones bucales y su impacto en la condición sistémica de los pacientes hospitalizados. En este trabajo se evaluó el conocimiento de los estudiantes de Odontología de MANAUS / AM sobre el papel de los odontólogos en un entorno hospitalario. Se trata de un estudio observacional, transversal, desarrollado de enero a marzo de 2020. El estudio incluyó a 80 estudiantes, matriculados habitualmente entre el 1er y el 8vo período, que estaban dispuestos a participar voluntariamente en el estudio y responder el cuestionario, después de leer y firmar. El formulario de consentimiento informado (TCLE). Esta investigación fue aprobada por el CEP dictamen CAAE: 28581020.6.0000.5512. Los resultados mostraron que el 50\% de los estudiantes nunca había vivido la experiencia en un ambiente hospitalario durante la carrera de Grado, aunque más de la mitad (64\%) de los entrevistados mostró interés por esta experiencia. Se encontró que el $41 \%$ cree que este contenido debe ser abordado en el Marco Curricular, a pesar de haber escuchado ya sobre el tema en las clases impartidas por un profesor de la asignatura. El 54\% de los estudiantes respondió que no se siente capaz de trabajar con un equipo médico y multidisciplinario en un entorno hospitalario, aunque el $65 \%$ de los estudiantes cree que el papel del odontólogo en este equipo es importante. Se concluye que, a pesar de la ausencia de la disciplina de Odontología Hospitalaria en la carrera de Grado en Odontología, existe interés en la gran mayoría de académicos por la odontología hospitalaria. Se sugiere implementar una disciplina en el plan de estudios centrada en la odontología hospitalaria.

Palabras chave: Atención odontológica; Personal de odontología em hospital; Estudiantes de odontología; Enseñanza.

\section{Introdução}

Pacientes internados em Unidade de Terapia Intensiva (UTI) muitas vezes se encontram totalmente dependentes de cuidados, portanto, impossibilitados de manter uma higienização bucal adequada, necessitando do suporte de profissionais da saúde para esta e outros tipos de tarefas. A aquisição e manutenção da saúde bucal, além de uma maior integração da Odontologia e da Medicina visando o tratamento global dos pacientes, se fazem necessárias (Rabelo et al., 2010; Blum et al., 2018).

A dificuldade de autocuidado do paciente em UTI é fator predisponente a instalação de patologias bucais, bem como a redução de fluxo salivar. Biofilme, cálculo dental, doença periodontal, lesões cariosas, necrose pulpar e traumas provocados por próteses podem influenciar na condição sistêmica do paciente e agravar seu quadro clínico. (Meire et al., 2010)

A bacteremia de etiologia oral também é comum em pacientes de UTI e importante causa de mortalidade. Sendo assim, a higiene oral nesses pacientes é essencial para reduzir a incidência de bacteremias e pneumonia por aspiração mecânica (Sarin, 2008). Com a aspiração de secreções na cavidade oral, reduz-se o risco de aspiração de microrganismos (Alhazzani et al., 2013; Simões et al., 2020).

A presença de um cirurgião dentista (CD) em âmbito hospitalar, é importante como suporte no diagnóstico das alterações bucais e como coadjuvante na terapêutica médica; seja na atuação em procedimentos emergenciais frente aos traumas; em procedimentos preventivos para se evitar o agravamento da condição sistêmica ou o surgimento de uma infecção hospitalar, e procedimentos curativos e restauradores para adequação do meio bucal e prover maior conforto ao paciente (Ferreira et al., 2017).

Apesar desta importância, há uma lacuna na matriz curricular dos cursos de Odontologia do Brasil no que diz respeito à possibilidade de atuação em ambiente hospitalar e UTI. Os futuros profissionais deveriam ter acesso e vivência neste ambiente, a fim de compreender o atendimento a pacientes sistemicamente comprometidos; participar da tomada de decisão sobre o diagnóstico, prognóstico e plano de tratamento do paciente, juntamente com a equipe multiprofissional atuante no hospital/UTI (Couto-Souza et al., 2021). 
É importante e se faz necessário que os cursos de Odontologia abordem este tema na Graduação e que este conteúdo faça parte da grade curricular seja através de disciplina eletiva ou optativa. Durante a formação acadêmica, o graduando em Odontologia deve ser incentivado e preparado para o manejo do paciente crítico seja em ambiente hospitalar ou em UTI. O resultado desta falha na formação acadêmica, é que muitos profissionais da área não sabem quais as atribuições de um cirurgião-dentista neste ambiente, e acabam por acreditar que só o Cirurgião Bucomaxilo-facial (CBMF), além de ter maior acesso aos hospitais, tem formação e está apto ao acompanhamento e tratamento de pacientes graves e críticos (Wayana et al., 2014; Medeiros et al., 2020).

Sendo assim, este trabalho se propôs a avaliar o conhecimento de acadêmicos de um curso de Odontologia em Manaus/AM, sobre a atuação do cirurgião-dentista em Unidade de Terapia Intensiva e hospitalar.

\section{Metodologia}

Trata-se de um estudo do tipo transversal, descritivo e quantitativo (Estrela, 2018), desenvolvido no período de janeiro a março de 2020. Participaram desta pesquisa, acadêmicos do $1^{\circ}$ ao $8^{\circ}$ períodos do curso de Odontologia de uma universidade em MANAUS/AM.

Foram incluídos na pesquisa todos os alunos que estavam regularmente matriculados durante o período do estudo e que se dispuseram a participar voluntariamente, após a leitura e assinatura do Termo de Consentimento Livre e Esclarecido (TCLE). 'O trabalho foi submetido e aprovado pelo Comitê de Ética (CEP) pelo CAAE:28581020.6.0000.5512.

Os dados foram coletados através da aplicação de um questionário contendo 13 questões, adaptado ao proposto por Pereira et al., (2021). O objetivo foi avaliar o conhecimento dos acadêmicos do curso de Odontologia a respeito da atuação do cirurgião-dentista no contexto hospitalar e o ensino deste conteúdo durante a graduação.

Os programas Microsoft Excel (Microsoft Office) e BIOESTAT 3.0 foram utilizados para tabulação e análise dos dados.

\section{Resultados}

A Tabela 1 apresenta os dados sociodemográficos dos participantes da pesquisa. Do total de 80 alunos que participaram do estudo, $68 \%(\mathrm{n}=54)$ eram do gênero feminino e a maioria $(38 \%, \mathrm{n}=30)$ cursavam o $7^{\circ}$ e $8^{\circ}$ períodos. A faixa etária predominante estava entre 17 e 23 anos.

Tabela 1 - Dados Sociodemográficos.

\begin{tabular}{lll}
\hline Dados Gerais & n & \% \\
\hline Gênero & 54 & 67,5 \\
Feminino & 26 & 32,5 \\
Masculino & 80 & 100 \\
Total & & \\
& & \\
Faixa etária & 62 & 77,5 \\
18 a 23 & 13 & 16,3 \\
23 a 28 & 5 & 6,2 \\
28 ou mais & 80 & 100 \\
Total & & \\
& & \\
Período letivo em curso & 29 & 36 \\
$1^{\circ}$ ao $3^{\circ}$ períodos & 21 & 26 \\
$4^{\circ}$ ao $6^{\circ}$ períodos & 30 & 38 \\
$7^{\circ}$ e $8^{\circ}$ períodos & $\mathbf{8 0}$ & $\mathbf{1 0 0}$ \\
Total & & \\
\hline
\end{tabular}

Fonte: Autores. 
O Gráfico 1 apresenta o questionamento ao acadêmico sobre a oportunidade de vivência odontológica em um hospital, em algum momento do curso de Graduação. Observamos que 50\% $(n=40)$ dos acadêmicos nunca vivenciaram esta experiência, enquanto $9 \%(\mathrm{n}=7)$ não tem interesse em ter uma experiência odontológica em hospitais.

Gráfico 1 - Você já teve alguma vivência odontológica em um hospital?

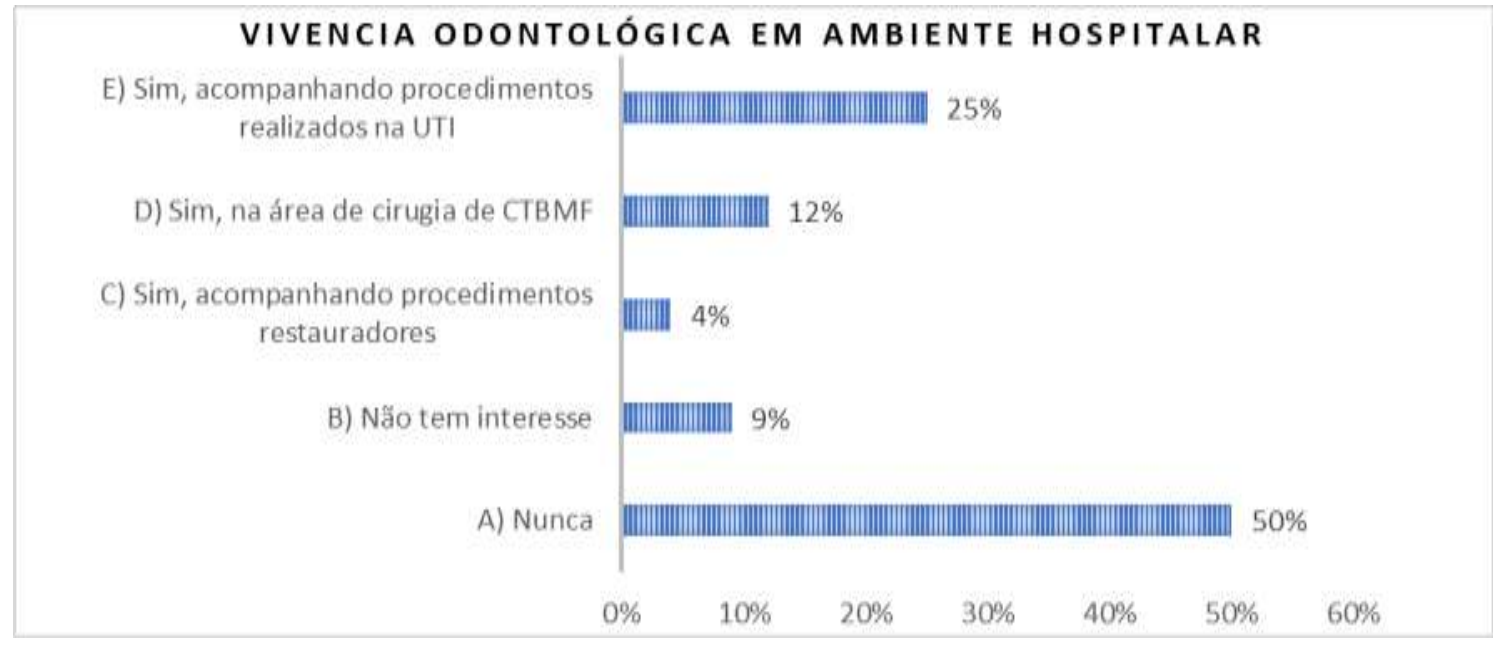

Fonte: Autores.

O interesse do acadêmico em vivenciar alguma experiência odontológica hospitalar, foi avaliado no Gráfico 2 . Observou-se que um pouco mais da metade (64\%) dos entrevistados possuem o interesse em vivenciar uma experiência em ambiente hospitalar por entenderem a presença do cirurgião-dentista é importante na equipe multiprofissional. No entanto, chama a atenção o fato de $11 \%(\mathrm{n}=9)$, acreditam que não seja importante a presença do cirurgião-dentista em ambiente hospitalar.

Gráfico 2 - Interesse do acadêmico de Odontologia pela vivência em ambiente hospitalar.

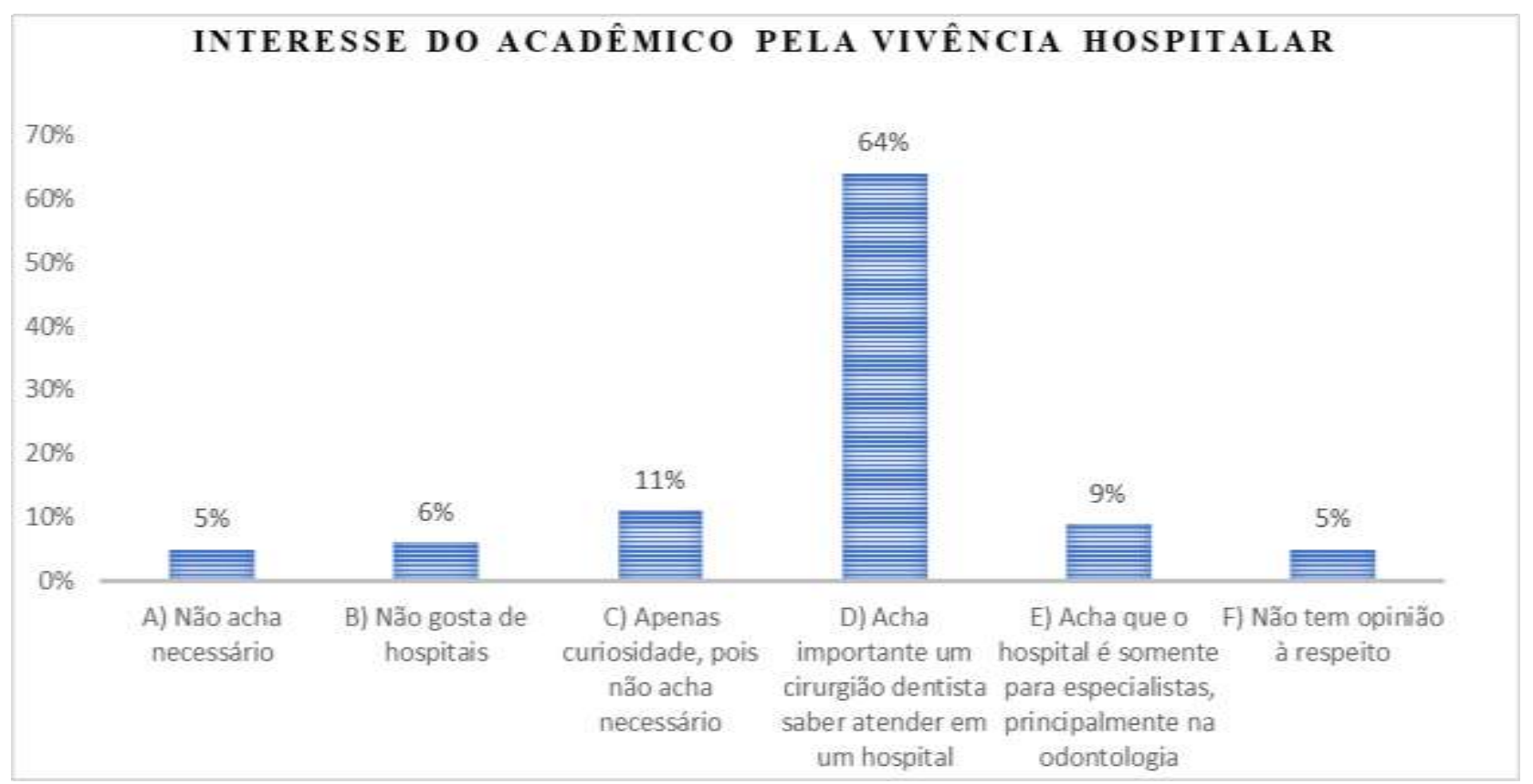

Fonte: Autores. 
Quando questionados sobre qual a especialidade é necessária e capacita para atendimento em ambiente hospitalar e UTIs, a maioria dos acadêmicos (35\%) acreditam que seja a especialidade Bucomaxilo-facial, seguida da Odontologia para Pacientes - PNE (19\%). Nenhum aluno respondeu sobre a Habilitação em Odontologia Hospitalar, regulamentada pelo CFO (Conselho Federal de Odontologia, que capacita o cirurgião-dentista para esta área (Gráfico 3).

Gráfico 3 - Especialidade necessária para atendimento em ambiente hospitalar - Visão do acadêmico.

\section{ESPECIALIDADE QUE CAPACITA PARA O ATENDIMENTO HOSPITALAR}

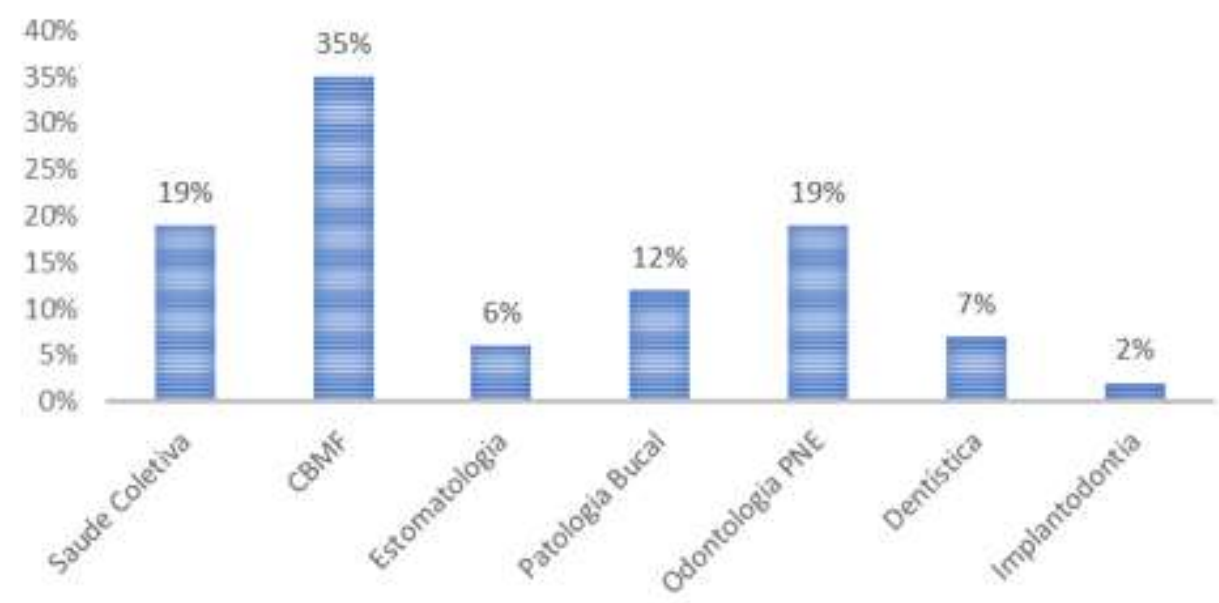

Fonte: Autores.

O Gráfico 4 aponta a opinião dos acadêmicos sobre a inserção da odontologia hospitalar como parte integrante da Grade curricular da graduação. $67 \%(\mathrm{n}=54)$ dos acadêmicos responderam este tema não faz parte do conteúdo programático de nenhuma disciplina, apesar de já terem ouvido falar sobre o assunto em aulas ministradas por docente do curso de Graduação.

Gráfico 4 - Opinião dos acadêmicos sobre a inserção da Odontologia Hospitalar na Grade Curricular do curso de Odontologia.

O TEMA ODONTOLOGIA HOSPITALAR É ABORDADO NA GRADE CURRICULAR DO CURSO?

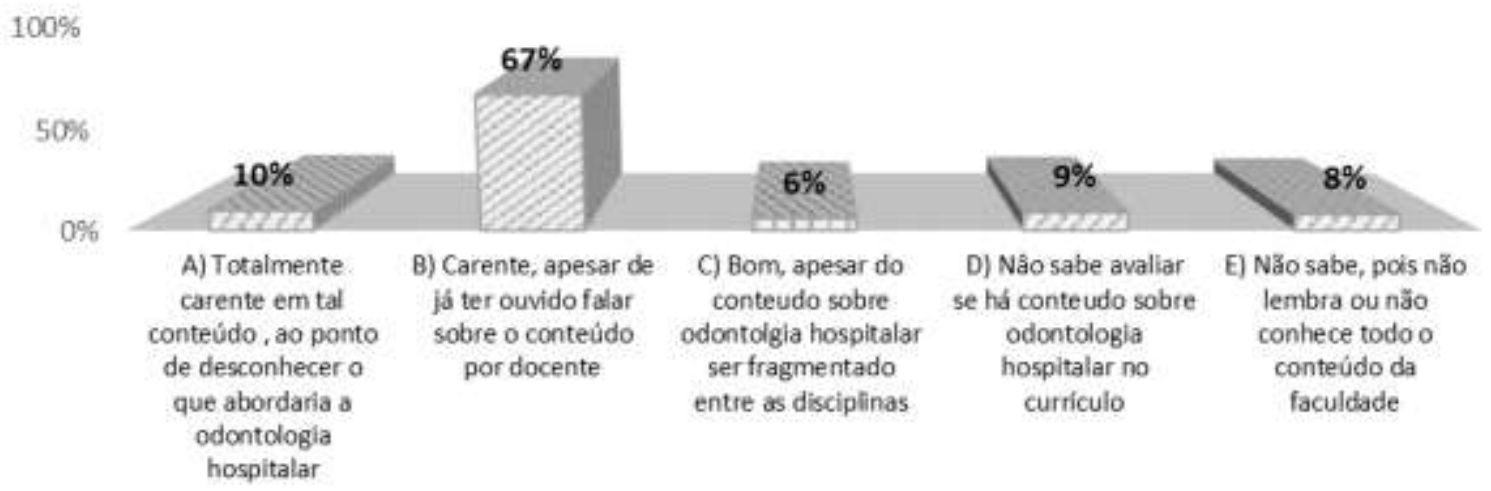

Fonte: Autores. 
Na opinião dos acadêmicos este assunto (Odontologia Hospitalar) deveria ser abordado no curso de graduação, em disciplinas onde os conteúdos aulas possibilitavam atendimento odontológico em atendimento hospitalar. 40\% ( $\mathrm{n}=32)$ apontaram que deveria ser na disciplina de Cirurgia Bucomaxilo-Facial e 34\% (n=27) em Traumatologia (Gráfico 5).

Gráfico 5 - Disciplinas que deveriam abordar o tema sobre Odontologia Hospitalar.

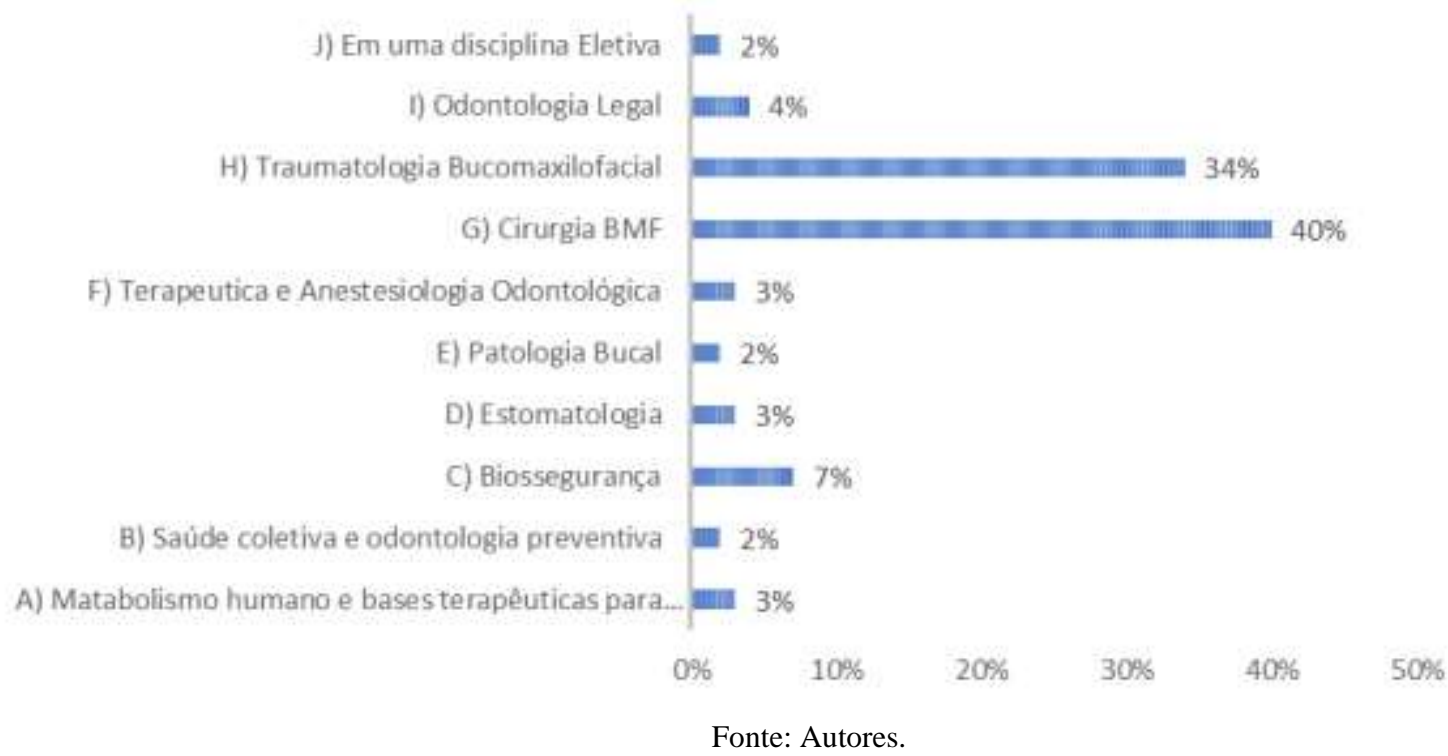

O Gráfico 6 aborda a visão do acadêmico sobre as atribuições do cirurgião-dentista em ambiente hospitalar. Observou-se que a maioria dos acadêmicos, $24 \%(\mathrm{n}=19)$ responderam que a atuação se dá no tratamento de traumas faciais, outros $23 \%(\mathrm{n}=18)$ acreditam que seja no diagnóstico de lesões bucais. E $15 \%(\mathrm{n}=12)$ responderam que no ambiente hospitalar o cirurgião-dentista atua somente no atendimento de emergências odontológicas.

Gráfico 6 - Atribuições do cirurgião-dentista no ambiente hospitalar, na visão do acadêmico.

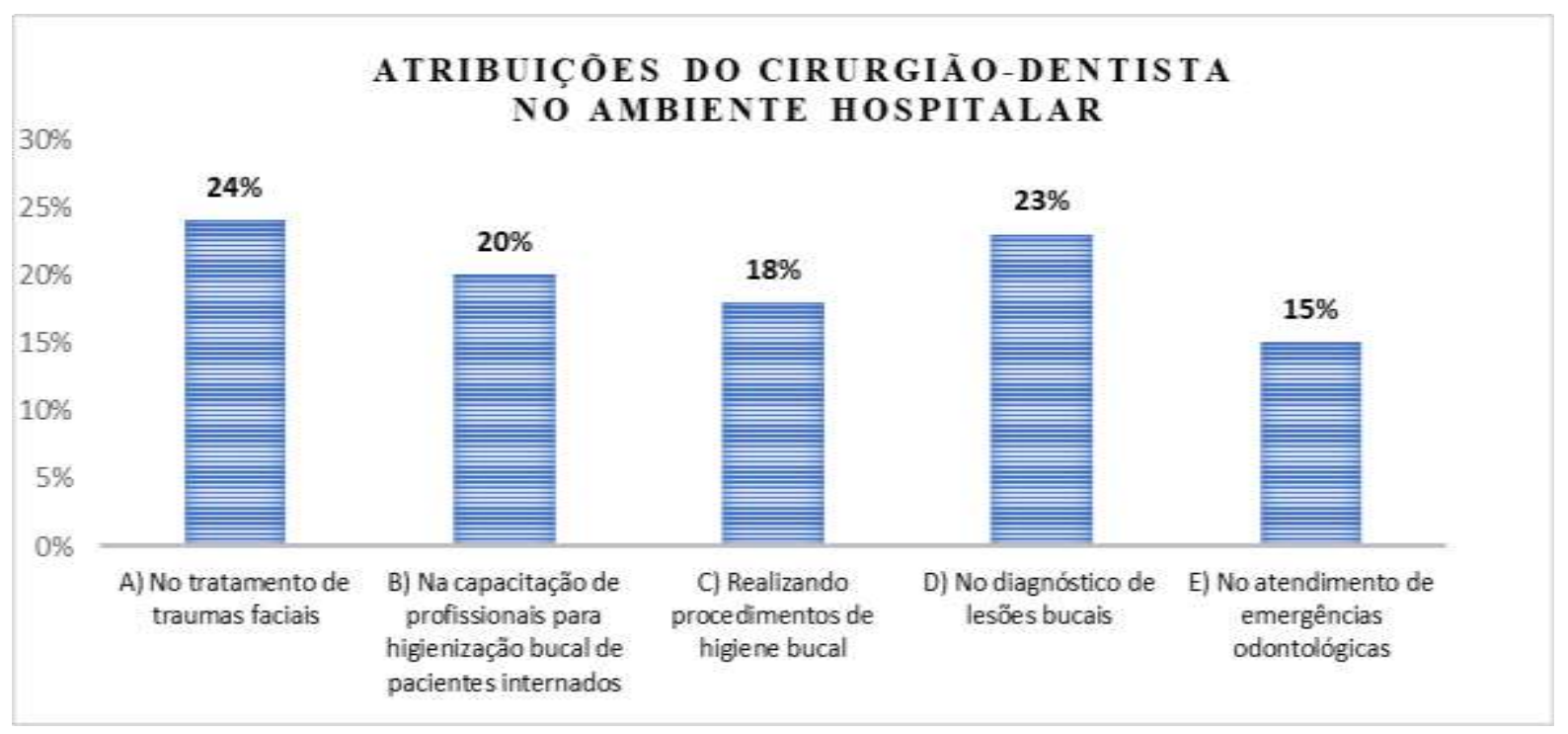

Fonte: Autores.

No Gráfico 7 observamos que 54\% $(n=43)$ dos acadêmicos responderam que não se sentem capacitados e aptos para trabalhar junto a uma equipe médica e multiprofissional em ambiente hospitalar. Enquanto 7\% (n=6) responderam que não acha necessário trabalhar junto a uma equipe médica em um hospital. 


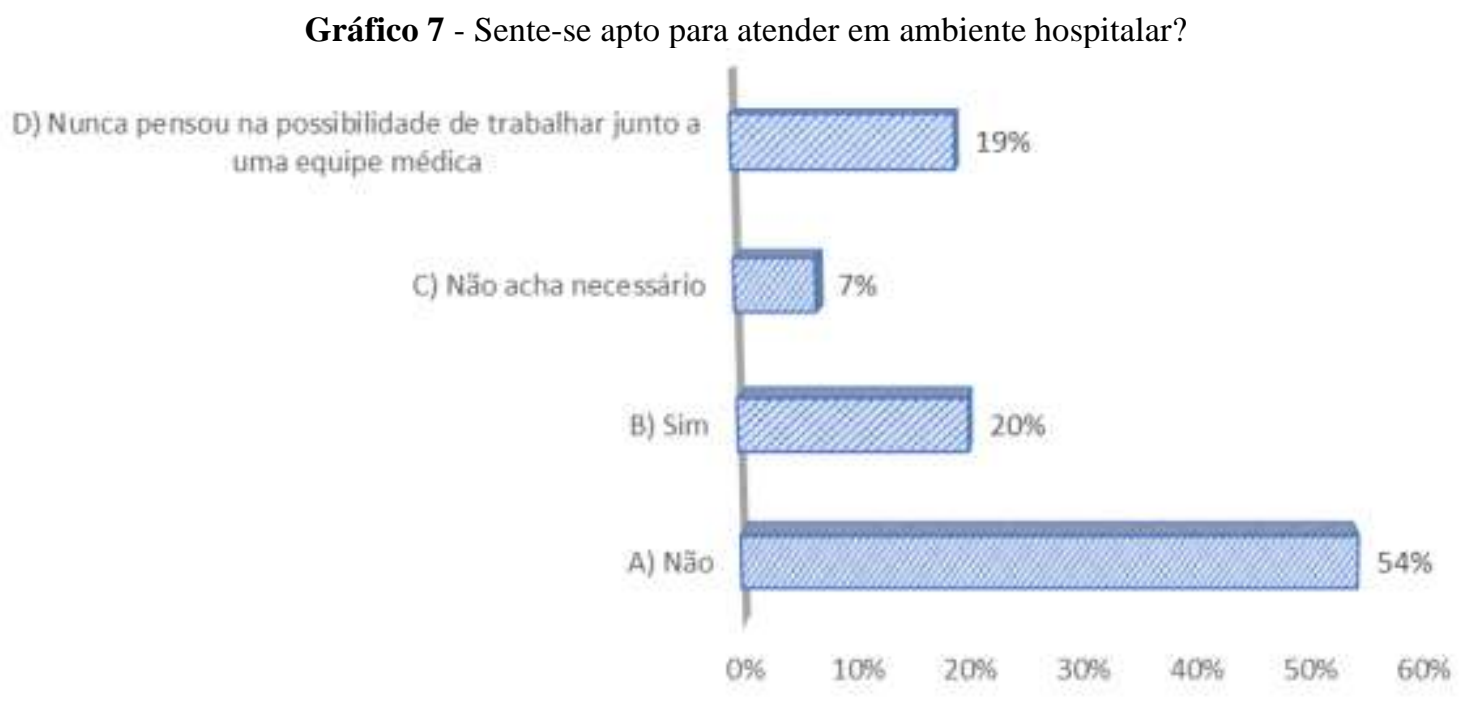

Fonte: Autores.

\section{Discussão}

Embora a ANVISA desde 2010, através da RDC 7, já tenha evidenciado que um dos requisitos mínimos para funcionamento das UTIs, é a assistência odontológica (RCD 7/2010, art. 18. §VI), muitos gestores municipais ou estaduais, ainda não instituíram a obrigatoriedade do cirurgião-dentista em ambiente hospitalar, embora a presença do Cirurgião Bucomaxilo-facial, seja uma realidade em muitos hospitais referência em trauma. Alguns estados como Minas Gerais, São Paulo, Mato Grosso do Sul e Distrito Federal, Alagoas, São Luiz do Maranhão e Santa Catarina já criaram suas próprias leis, que tornam obrigatória a presença do cirurgião-dentista e atendimento odontológico em ambiente hospitalar (Silva et al., 2020).

Corroborando com a resolução da ANVISA, o Conselho Federal de Odontologia (CFO), através do Código de Ética Odontológica (CEO) (Brasil, 2012), no artigo 18, capítulo IX, descreve que "compete ao cirurgião-dentista a internação e assistência ao paciente, em hospitais públicos e privados, com e sem caráter filantrópica". Fica claro então que a odontologia hospitalar $(\mathrm{OH})$ é uma área de atuação do cirurgião-dentista, que apresenta respaldo conforme descrito.

Em 2015, o Conselho Federal de Odontologia (Brasil, 2015), através da Resolução 162/2015, regulamentou a prática da $\mathrm{OH}$ pelo cirurgião-dentista. Apesar de já autorizado e ser necessário, o atendimento odontológico nas Unidades de Terapia Intensiva (UTIs) e ambiente hospitalar é uma realidade em poucos hospitais brasileiros.

A presença do odontólogo em UTI ou em hospitais, tem o objetivo de prevenir não só as infecções bucais que interferem na evolução das doenças sistêmicas dos pacientes acamados, bem como limitar a disseminação de micro-organismos que colonizam desde a cavidade oral ao trato respiratório inferior desses pacientes. Os cuidados com a saúde bucal podem diminuir o tempo de internação do paciente e os custos hospitalares (Assis, 2012; Blum et al., 2018).

Os estudos mostram claramente que a quantidade de biofilme bucal em pacientes de UTI aumenta com o tempo de internação, paralelamente também ocorre aumento de patógenos respiratórios que colonizam o biofilme bucal, sendo esse um reservatório importante de patógenos. Neste sentido, se faz necessário à aquisição e manutenção da saúde bucal, além de maior integração da Odontologia e da Medicina, visando o tratamento global dos pacientes, a prevenção de doenças e maior humanização dos pacientes internados em UTI (Morais et al., 2006; Miranda et al., 2018).

E mesmo com tantos benefícios sobre o atendimento odontológico hospitalar, um outro agravante é a ausência da abordagem sobre as atividades e atuação odontológica nos hospitais, durante o curso de Graduação. Esta lacuna pode gerar 
algumas consequências tais como a falta de interesse pela área, ausência de interação com equipe multiprofissional e assistência odontológica aos pacientes críticos.

Acaba de ser publicado muito recentemente as Diretrizes Curriculares Nacionais (DCN) para o Curso de Odontologia (Brasil, 2021), através da Resolução $N^{\circ}$ 3, de 21 de junho de 2021. A Seção I trata dos Conteúdos Curriculares do curso e específica no artigo 25 parágrafo XI, que "Nas Ciências Odontológicas, incluem-se os conteúdos teóricos e práticos para compreensão e domínio: da assistência odontológica a indivíduos mantidos em Instituições de Saúde, incluindo ambientes hospitalares".

Esta nova proposta da DCN, acima citada, traz uma luz ao questionamento de vários autores (Wayana et al., 2014; Lucas et al., 2017; Medeiros et al., 2020; Couto-Souza et al., 2021) sobre ausência na grade curricular do tema: Odontologia Hospitalar e as possibilidades de atuação do cirurgião-dentista. Os resultados do presente estudo estão de acordo com o descrito pelos autores citados e descrevem que mais da metade dos alunos (67\%) apontaram que há carência deste conteúdo na Graduação e 54\% se dizem inaptos a atender em ambiente hospitalar e UTI. Estes resultados podem ser um reflexo da falta de abordagem e vivência em ambiente hospitalar pelos graduandos, embora o tema já tenha sido abordado por docentes do curso.

Sobre a inserção do tema na Matriz Curricular do curso de Odontologia, as DCN do curso de Odontologia, foram reformuladas recentemente em junho de 2021 e propõe na Seção III sobre a flexibilidade curricular. O Art. 29 postula que: " $O$ currículo do curso de graduação em Odontologia deverá incluir elementos que considerem a inserção institucional do curso, as demandas e as expectativas de desenvolvimento do setor de saúde na região no qual ele se insere, bem como a flexibilidade individual de estudos, de forma a permitir distintos percursos formativos para os estudantes" (Brasil, 2021). Cabe à Instituição de Ensino Superior (IES), adequar o Projeto Pedagógico do Curso (PPC), a esta nova proposta da DCN. A inserção da Odontologia Hospitalar poderia ser incorporada no Estágio Curricular Obrigatório ou na disciplina de Cirurgia Avançada. O objetivo é que o acadêmico poderia acompanhe um CD que já atue em ambiente hospitalar e que de preferência seja docente do curso de Odontologia, o que facilitaria a conexão entre os conteúdos abordados na aula teórica, com a vivência clínica (Couto-Souza et al., 2021).

As vantagens desta nova abordagem seriam inúmeras e dentre elas podemos citar: futuros profissionais mais seguros em relação ao atendimento clinico de pacientes sistemicamente comprometidos, o contato com a equipe multiprofissional atuante em UTI, tomada de decisão e conduta sobre a melhor conduta ao paciente crítico juntamente com a equipe médica, possibilidade de orientar a equipe de Enfermagem sobre a técnica de Higiene Oral com a finalidade de prevenir Pneumonia Nosocomial e por fim, uma formação mais completa e com o conhecimento teórico-prático de mais uma área de atuação e consequentemente mais profissionais aptos a atuar em UTI e Hospitais.

\section{Conclusão}

O presente estudo nos mostra que os acadêmicos do curso de Odontologia da IES em questão, em sua maioria, relatam que a grade curricular do curso de Graduação não contempla o conteúdo sobre Odontologia Hospitalar e UTI, e que gostariam de vivenciar este tipo de experiência. Sendo assim, torna-se evidente a necessidade de adequação do PPC e matriz curricular do curso de Graduação em Odontologia da maioria das Universidades do Brasil, para que contemple esta prática. Há carência de profissionais nesta área e apresentar ao estudante de Odontologia este novo cenário irá de fato preparar o futuro profissional para atuar neste ambiente e consequentemente maior competência para atender pacientes sistemicamente comprometidos no consultório odontológico. Sugerimos estudos futuros que abordem a inserção da Odontologia Hospitalar na grade curricular dos cursos de Odontologia do norte do Brasil. 


\section{Referências}

Alhazzani, W., Smith, O., Muscedere, J., Medd, J., \& Cook, D. (2013). Toothbrushing for Critically Ill Mechanically Ventilated Patients: A Systematic Review and Meta-Analysis of Randomized Trials Evaluating Ventilator-Associated Pneumonia. Critical Care Medicine, 41 (2): $646-55$.

Almeida, T. H. A. (2019). A atuação do Cirurgião Dentista na equipe multidisciplinar em Unidade de Terapia Intensiva: a visão do acadêmico. 50f. Monografia (Curso de Odontologia) - Centro Universitário de João Pessoa - UNIPÊ.

Assis, C. (2012). Atendimento Odontológico nas UTIs. Revista Brasileira de Odontologia, 69 (1): 72-5.

Blum, D. F., Silva, J. A., Baeder, F. M., \& Della Bona, A. A. (2018). Atuação da Odontologia em unidades de terapia intensiva no Brasil. Revista Brasileira de Terapia Intensiva. 30(3):327-332.

Brasil. (2012). Conselho Federal de Odontologia. Código de Ética Odontológica. Aprovado pela Resolução CFO-118/2012. Brasília, CFO 2012. https://website.cfo.org.br/wp-content/uploads/2018/03/codigo etica.pdf.

Brasil. (2015). Conselho Federal de Odontologia. Resolução CFO-162/2015. https://website.cfo.org.br/wp-content/uploads/2015/12/ResolucaoCFO-16215.pdf.

Brasil. (2021). Diário Oficial Da União. Resolução $N^{o}$ 3/2021. Institui as Diretrizes Curriculares Nacionais do curso de graduação em Odontologia e dá outras providências. Edição112, Seção 1, Página 69. Ministério da Educação. https://www.in.gov.br/web/dou/-/resolucao-n-3-de-21-de-junho-de-2021-327321299. Acesso em 30/06/2021.

Brasil. (2010). Ministério da Saúde. Agência Nacional de Vigilância Sanitária. RDC no 7 de 24 de fevereiro de 2010. Dispõe sobre os requisitos mínimos para funcionamento de Unidades de Terapia Intensiva e dá outras providências. Diário Oficial República Federativa do Brasil, Poder Executivo, Brasília, 24 fev. 2010 .

Couto-Souza, P. H., Friedlander, A. H., \& Berti-Couto, S. A. (2021). A ausência da formação hospitalar no currículo dos cursos de graduação em Odontologia é um motivo de preocupação. Revista da ABENO, 2021; 21(1), 1189.

Estrela, C. (2018). Metodologia científica: ciência, ensino, pesquisa. (3a ed.), Artes Médicas.

Ferreira, J. A., Londe, L. P., \& Miranda, A. F. (2017). A Relevância do Cirurgião-Dentista na UTI: Educação, Prevenção e Mínima Intervenção. Revista Ciência e Odontologia, 1(1):18-23.

Marín, C., Lanau, C. G., \& Bottan, E. R. (2016). A perspectiva de estudantes do curso de odontologia sobre a atuação do cirurgião-dentista em ambiente hospitalar. Revista Unimontes Cientifica, 18(2):1-7.

Meira, S. C. R., Oliveira, C. A. S., \& Ramos, I. J. M. (2010). A importância da participação do cirurgião-dentista na equipe multiprofissional hospitalar. $9^{a}$ edição do prêmio SINOG de Odontologia. Curso de Odontologia do Centro Universitário Newton Paiva, Belo Horizonte/MG.

Medeiros, Y. L., Faria, L. V., Lopes, D. F., de Oliveira, I. S., Fabria, G. M. C. (2020). Inserção da Odontologia Hospitalar na grade curricular dos cursos de Odontologia do sudeste brasileiro. Revista da Faculdade de Odontologia de Porto Alegre, 61(1):85-91.

Miranda, A. F., El Haje, G. L. C., de Paula, R. M., Costa, P. P., Biazevic, M. G. H. (2018). Assessing the incidence of tongue coating in patients treated in intensive care units. Revista Brasileira de Odontologia, 75(e1213):1-5.

Morais, T. M. N., Silva, A., Avi, A. L. R. O., Souza, P. H. R., Knobel, E., Camargo, L. F. A. (2006). A importância da atuação odontológica em pacientes internados em unidade de terapia intensiva. Revista Brasileira de Terapia Intensiva, 18(4): 412-417.

Rabelo, G. D., Queiroz, C. I., \& Santos, P. S. S. (2010). Atendimento odontológico ao paciente em unidade de terapia intensiva. Arquivos médicos dos hospitais e da Faculdade de Ciências Médicas da Santa Casa de São Paulo, 55(2): 67-70.

Sarin, J., Balasubramaniam, R., Corcoran, A. M., Laudenbach, J. M., Stoopler, E. T. (2008). Reducing the Risk of Aspiration Pneumonia among Elderly Patients in Long-Term Care Facilities through. Journal of the American Medical Directors Association, 9 (2): $128-35$.

Silva, G. E. M., Thomsen, L. P. R., Lacerda, J. C. T., Botelho, S. H. B., Reis, J. A. C., Ferreira, R. D. A., Resende, R. G. (2020). Odontologia hospitalar no Brasil: onde estamos? Uma análise do cenário dos últimos anos. Revista da Faculdade de Odontologia de Porto Alegre, 61(1): 92-97.

Simões, T. M. S., Fernandes Neto, J. de A., Ferreira, A. C. D., Sousa, J. A., de Medeiros, C. L. S. G., Catão, M. H. C. V. (2020). Controle do biofilme oral e sua relação com a redução de infecções respiratórias em pacientes de UTI: uma revisão de ensaios clínicos. Research, Society and Development, 9(10): e8339108594.

Wayama, M. T., Aranega, A. M., Bassi, A. N. F., Ponzoni, D., Garcia Junior, I. R. G. (2014). Grau de conhecimento dos cirurgiões-dentistas sobre Odontologia Hospitalar. Revista Brasileira de Odontologia, 71(1): 48-52. 